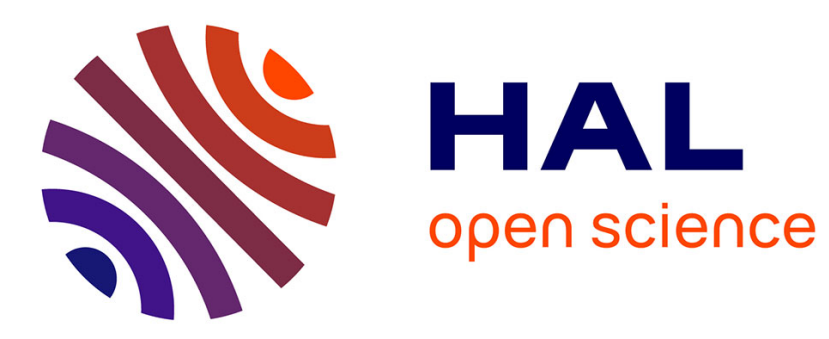

\title{
Analysis of Energy and Cost Savings in Hybrid Base Stations Power Configurations
}

\author{
Ali El Amine, Hussein Al Haj Hassan, Loutfi Nuaymi
}

\section{To cite this version:}

Ali El Amine, Hussein Al Haj Hassan, Loutfi Nuaymi. Analysis of Energy and Cost Savings in Hybrid Base Stations Power Configurations. VTC 2018 - IEEE 87th Vehicular Technology Conference, Jun 2018, Porto, Portugal. 10.1109/VTCSpring.2018.8417652 . hal-01923392

HAL Id: hal-01923392 https://imt-atlantique.hal.science/hal-01923392

Submitted on 28 Nov 2018

HAL is a multi-disciplinary open access archive for the deposit and dissemination of scientific research documents, whether they are published or not. The documents may come from teaching and research institutions in France or abroad, or from public or private research centers.
L'archive ouverte pluridisciplinaire HAL, est destinée au dépôt et à la diffusion de documents scientifiques de niveau recherche, publiés ou non, émanant des établissements d'enseignement et de recherche français ou étrangers, des laboratoires publics ou privés. 


\title{
Analysis of Energy and Cost Savings in Hybrid Base Stations Power Configurations
}

\author{
Ali El-Amine ${ }^{1}$, Hussein Al Haj Hassan ${ }^{2}$, Loutfi Nuaymi ${ }^{1}$ \\ ${ }^{1}$ Departement Système Réseaux, Cybersécurité et Droit du numérique (SRCD). IMT-Atlantique. Rennes. France \\ ${ }^{2}$ Faculty of Engineering. Lebanese University. Beirut. Lebanon \\ Email: ${ }^{1}$ firstname.lastname@imt-atlantique.fr
}

\begin{abstract}
Wireless networks have important energy needs. Many benefits are expected when the base stations, the fundamental part of this energy consumption, are equipped with renewable energy (RE) systems. Important research efforts have been done to enhance the utilization of RE. However, to the best of our knowledge, these efforts did not take into consideration partially RE-equipped systems. The latter is of great importance considering the high cost of these systems and the feasibility of implementing RE systems at all base station sites. Thus, it is interesting to study the percentage of sites to be equipped with RE systems. In this work, we analyze the energy and cost savings for a defined energy management strategy of a RE hybrid system. Our study of the relationship between cost savings and percentage of sites equipped with RE show significant results. For example, our simulation shows that a cost gain of $60 \%$ is realized when $30 \%$ of the base stations are equipped with solar panels that harvest only $35 \%$ of the total network energy demand at full load. Results also show an upper limit for the battery capacity at which the cost gain is maximized.
\end{abstract}

\section{INTRODUCTION}

The world of wireless communication is gaining popularity due to its ongoing advances towards new services and features that were implausible in the past. Nevertheless, this growing popularity is significantly increasing the user traffic and hence, proliferating the energy consumption of the Information and Communication Technology (ICT) sector. It is estimated that ICT consumes around $4.7 \%$ of the world's electrical energy, releasing into the atmosphere about $1.7 \%$ of the global $\mathrm{CO}_{2}$ emissions [1]. Furthermore, mobile operators are among the top energy consumers with a contribution share of $0.5 \%$ of the total energy supply worldwide [2]. This number is ought to increase with the fast growth in capacity demand, unless compelling enhancements are brought to network's efficiency. In its recent VNI mobile traffic report, Cisco forecasts an increase of sevenfold in the global mobile data traffic volume between 2016 and 2021 [3].

In 3G and LTE cellular networks, Radio Access Network (RAN) consumes the major part of energy with the base station (BS) using 75-80\% of the network's energy [4]. Hence, reducing the power at this level is important for the energy efficiency of cellular networks. Several techniques attack the aforementioned challenge on different layers: Cell Layout Adaptation (CLA) layer, including cell breathing, switching-off techniques, and the deployment of small-cells/relays; Radio Resource Management (RRM) layer, including transmission power control and the optimization of transmission resources in time or bandwidth; Environmental Learning and Information Exchange (EL-IE) layer, such as cognitive radio, to optimize spectrum efficiency and energy consumption; and component level enhancement layer, including hardware components efficiency such as, power amplifier (PA) [5]. Moreover, providing energy harvesting capability to cellular BSs is another effective approach for an energy efficient network, to acquire clean and cheap energy from ambient surrounding. A detailed survey on powering cellular BSs with renewable energy (RE) can be found in [6].

In the context of powering cellular BSs with local RE sources and due to the high cost of these systems, careful study of RE sizing and deployment is needed to avoid additional CAPEX and OPEX costs, and to ensure a good quality of service (QoS) to the offered users. For instance, deploying $\mathrm{RE}$ on all sites of a cellular network might not be a feasible economic solution for the operator. In contrast to small scale systems that focus on maximizing the throughput for point to point links powered by RE, this paper studies the network on a large scale and focuses on the design and operation of wireless cellular networks powered with hybrid energy supplies (RE and smart grid). In particular, we focus on studying the impact of equipping sites with RE sources on the operational cost and the performance of a cellular network, to decide how much to invest in RE, i.e., number of sites equipped with RE sources, sizing of RE, and battery capacity, etc.

The rest of the paper is organized as follows: Section II presents a brief overview of the existing work. In Section III, we detail the system model. We describe the online strategies and policies used for cost reduction in Section IV. Finally, we present the simulation results in Section V before concluding in Section VI.

\section{RELATED WORK}

The use of RE in cellular networks has received a number of studies. The idea is to optimize the use of $\mathrm{RE}$ in order to achieve the required objective(s), e.g., reduce the on-grid energy consumption and minimize the electric bill of the operator. 
Bringing down on-grid energy consumption in cellular networks has been thoroughly studied. For example, the authors in [7] exploited the approach of energy sharing and load shifting under the smart grid (SG) environment, with the objective to minimize the on-grid energy consumption of green cellular networks, powered with hybrid energy supplies (RE and SG). While the authors ignored sleep mode strategies, they assumed that the network is fully equipped with RE sources. In a similar network setup and objective, the authors in [8] studied the joint problem of sleep scheme approach and resource allocation taking into account the users' blocking probability as the QoS. Using dynamic programming (DP), their numerical results revealed a $50 \%$ reduction in grid power consumption compared with the BS on-off algorithm.

The objective of minimizing the electric bill of a cellular network operator was investigated in [9], [10], [11]. In [9], we studied RE allocation, energy consumption minimization, and radio resource allocation to minimize the electric bill of a cellular network powered by both $\mathrm{RE}$ and power grid, in a variable electricity price environment. In [10], the authors presented intelligent green energy allocation, user association, and downlink power control to increase the cost savings of cellular network operators taking into account, the network's delay. In [11], based on stochastic dynamic programming, the authors studied BS switch-off to reduce the total on-grid energy cost in a large-scale green cellular network.

Even though the above mentioned work succeeded in accomplishing the above objectives, the models studied assumed that all network's BSs are equipped with RE sources. This assumption, however, might not be practical for large networks of thousands of BSs. The closest work to our study is done in our previous work [12], where we evaluated the trade-off between the percentage of non-green energy used and the user satisfaction degree, that is defined as the User-BS coverage provided by hybrid energy powered BS. However, the work was limited to one BS only.

\section{SySTEM MODEL}

\section{A. Network Description}

We consider a geographical area served by a mobile operator deploying an LTE network with $M$ macro BSs. These BSs are located in a hexagonal structure with site distance of $\mathrm{D}$, serving $K$ mobile users. We denote the set of BSs by $\mathcal{B}=\{1, \ldots, j, \ldots, M\}$. A BS $j$ serves $k_{j}$ users such that, $\sum_{j=1}^{M} k_{j}=K$. Initially, the users are associated with and served by the BSs based on best signal-to-interference-plus-noise ratio method (Best SINR), that is managed by a centralized energy management unit (EMU). Each user measures the SINR by using pilot signals from all BSs and sends it to the EMU. We further consider two sets of BSs. One is solely powered by grid energy, i.e., smart grid (SG) and is denoted by $B S^{\text {Grid }}$. The other set is equipped with RE and batteries; hence, it is powered by a mix of hybrid energy sources ( $\mathrm{SG}$ and RE) and is denoted by $B S^{M i x}$. The total bandwidth of the system $W$ is shared among the BSs with a frequency reuse factor of one, thus each BS is using the whole system bandwidth. We consider that the traffic load, on-grid energy price, and RE are all varying over time.

\section{B. Network Architecture and Operations}

Since equipping all sites with RE sources might not be economically feasible, we consider installing a percentage of sites with energy harvesters. Furthermore, we allow BSs to switch to sleep mode state temporarily to save energy. As shown in Fig. 1, a centralized EMU manages the operational states of a cluster of BSs. Each user sends all received SINRs from surrounding BSs to the EMU. Based on these measurements, the BSs are set to either active or sleep mode for a fixed period of time.

The decision of allocating RE, on the other hand, is left for each BS. Based on the current price of electricity and the state of charge of the battery, a local EMU installed at each BS, as shown in Fig. 1, decides whether to store or use the harvested energy. The decision policies for switching-off BSs and allocating RE are detailed in Section IV.

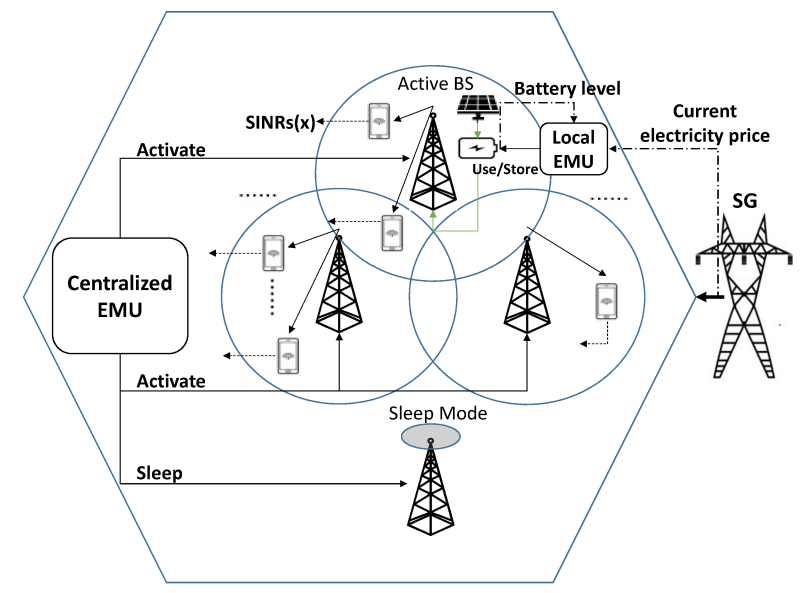

Fig. 1: Network architecture and operations.

\section{Energy Consumption Model}

In order to calculate the power consumption of the wireless cellular network, we use the power model provided by EARTH [13]. Accordingly, the power consumption of a BS consists of a static part $P_{0}$ and a traffic dependent part $P_{\text {out }}$. Moreover, the power demand depends on the number of active RBs. At time stage $i$, and because of the nearly linear relationship that exists between the RF power and the BS power consumption, the power consumption of the BS can be expressed as 
follows:

$$
\begin{aligned}
& P_{\text {in }, i}= \\
& \begin{cases}N_{\text {trx }}\left(P_{0}+\frac{n_{R B}^{(i)}}{n_{R B}^{T \text { otal }}} \times \Delta_{p} \times P_{\text {out }}^{(i)}\right), & 0<P_{\text {out }} \leq P_{\text {max }}, \\
N_{\text {trx }} P_{\text {sleep }}, & P_{\text {out }}=0 .\end{cases}
\end{aligned}
$$

where $N_{t r x}$ is the number of transceivers. $\Delta_{p}$ and $P_{\text {sleep }}$ are the load dependent parameter and the power consumption of the BS in sleep mode, respectively. $n_{R B}^{(i)}$ and $n_{R B}^{T o t a l}$ are the number of active RBs at time stage $i$ and the maximum number of RBs available to the BS, respectively.

\section{Downlink Transmission Model}

We measure the downlink transmission quality between a BS $j$ and a user located at a location $x$, denoted by $u_{x}$, based on the SINR as follows:

$$
\operatorname{SINR}_{j}\left(u_{x}\right)=\frac{P_{j} h_{j}\left(u_{x}\right)}{\sigma^{2}+\sum_{j^{\prime} \in \mathcal{B}, j^{\prime} \neq j} P_{j^{\prime}} h_{j^{\prime}}\left(u_{x}\right)}
$$

where $P_{j}$ is the transmitted power of BS $j, h_{j}\left(u_{x}\right)$ is the channel gain from BS $j$ to user at location $x$, which accounts for the path loss and shadowing effect, and $\sigma^{2}$ is the additive white Gaussian noise power density. A user is associated to the corresponding BS based on the highest received SINR.

We can express the rate offered to a user at position $x$ by BS $j$ using Shannon-Hartley theorem as follows:

$$
R_{j}\left(u_{x}\right)=n_{R B}^{\left(u_{x}\right)} \times B W_{R B} \times \log _{2}\left(1+S I N R_{j}\left(u_{x}\right)\right)
$$

where $n_{R B}^{\left(u_{x}\right)}$ is the number of RBs allocated to user at location $x$ and $B W_{R B}$ is the bandwidth of one RB, such that $B W_{R B}=W / n_{R B}^{T o t a l}$.

\section{E. Renewable Energy Generation, Electricity Price and Traffic Load Variations}

We consider the use of solar panels due to their flexibility. In addition, we provide these sites with battery storages in order to store the excess harvested energy for future transmission. The amount of harvested energy varies depending on the location and panel size. We adopt a panel size of $12.5 \mathrm{~m}^{2}$ and take as an example the city of Marseille (France), due to its high solar potential, $\left(7.08 \mathrm{KWh} / \mathrm{day} / \mathrm{m}^{2}\right)$ [14].

The traffic load on the other hand manifests both temporal and spatial diversities. On the temporal diversity, the traffic load of a BS dynamically changes over time, as shown in Figure 2. We assume that the users are uniformly distributed in the area, and the BSs are always transmitting data to the users.

We consider real-time pricing (RTP) to model the electricity price variations. This model is expected to dominate the market (particularly the industrial one), due to its advantages in reducing gas emission levels, load balancing, and the PAR (peak-to-average ratio) [15].

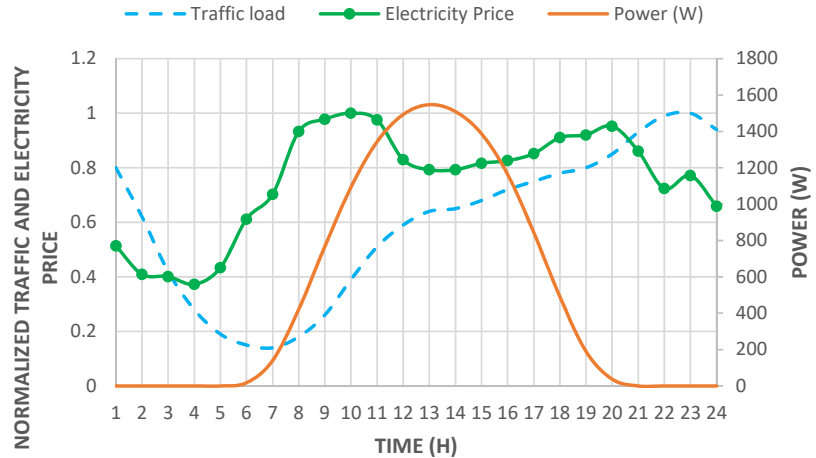

Fig. 2: RE power, traffic load and electricity price variations [13], [14], [16].

\section{ONLINE RAN ENERGY MANAGEMENT STRATEGY}

Several radio resource management techniques, sleeping scheme mechanisms, and battery power management algorithms were designed and studied to enhance the energy efficiency of wireless cellular networks [6]. In this section, we present some of these strategies and use them to analyze the system understudy.

\section{A. Sleep Mode Strategy: SINR threshold-based method}

The algorithm described in this section aggressively turns off BSs based on an SINR Switch-Off Threshold $\left(S I N R_{S O T}\right)$ parameter. It is a modified version of the sleep scheme algorithm presented in [9]. It considers the set of BSs powered solely by grid energy, $B S^{\text {Grid }}$, as candidates to be switched-off. First, it sorts these BSs in increasing order of their traffic loads. Then, starting with BS $j \in B S^{G r i d}$ with the lowest load, the algorithm switches it to sleep mode if the measured SINRs between each of its users and all neighboring BSs (except $j$ ) are above $S I N R_{S O T}$.

$$
\begin{aligned}
& S I N R_{j^{\prime}}\left(u_{x}\right) \geq S I N R_{S O T}, \forall u_{x} \in B S_{j}^{\text {Grid }} \text { and } \\
& j^{\prime} \in\{\mathcal{B} \backslash j\} .
\end{aligned}
$$

If (4) is satisfied, BS $j$ offloads its users to neighboring BSs with the highest received SINRs. The $S I N R_{S O T}$ parameter described in this section is nonoperational. In other words, the SINRs of the users offloaded to neighboring BSs will witness a signal improvement due to interference reduction, as a result of switching-off some BSs. The algorithm is summarized in Table I.

\section{B. Resource Block Policy: Max-Min Fairness algorithm}

We consider that each BS has a fixed number of total RBs $\left(R B_{T}\right)$ that is shared among its associated users. In this work, we take advantage of the Max-Min fairness 
TABLE I: Algorithm for sleep scheme: SINR threshold-based method.

\begin{tabular}{|c|c|}
\hline \multicolumn{2}{|c|}{ Algorithm 1: Sleep scheme algorithm } \\
\hline Define: & $\begin{array}{l}\text { BS-User association matrix: } \mathbf{A} \in \\
\mathbb{Z}_{2}^{K \times M} \text {; Users associated with BS } j \text { : } \\
\mathbf{U}_{j} \text {. }\end{array}$ \\
\hline Initialize: & $\begin{array}{l}\text { A based on Best SINR; } S I N R_{S O T} \text {. } \\
\text { Sort the BSs } \in B S^{G r i d} \text { in increasing } \\
\text { order of traffic loads. }\end{array}$ \\
\hline for $j=1: \mid H$ & $\begin{array}{l}\text { Grid } \\
\text { Switch off } \mathrm{BS} j \text { if (4) is satisfied. } \\
\text { Offload its users } \mathbf{U}_{j} \text { to neighboring } \\
\text { BSs with highest SINRs. }\end{array}$ \\
\hline Output: & $\begin{array}{l}\text { Updated BS-User association } \mathbf{A} \text { and } \\
\text { BSs modes (active or sleep). }\end{array}$ \\
\hline
\end{tabular}

algorithm, detailed in [17], to share the RBs among the users in order to satisfy their QoS requirements. We further turn off excess RBs to save energy. In our simulation, we obtained a $22 \%$ gain when using the MaxMin Fairness algorithm compared to the case where all the RBs are used. The algorithm is summarized in Table II.

TABLE II: Algorithm for resource block allocation.

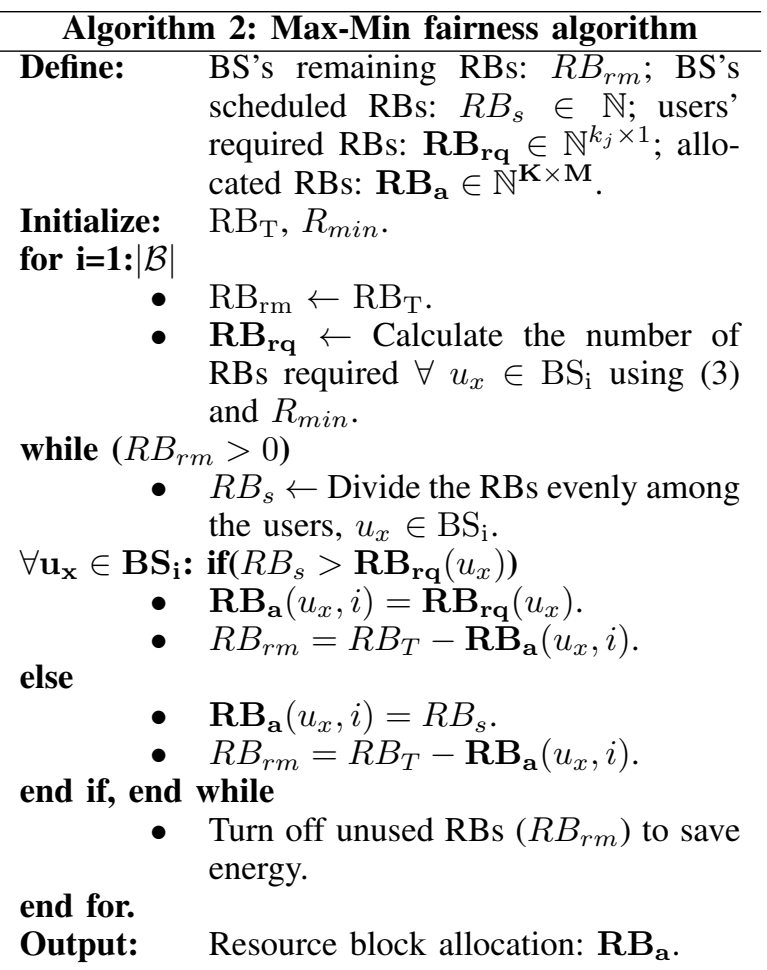

\section{Online Battery Management: SPAEMA algorithm}

Studies showed that intelligent energy management of harvested energy yields to higher on-grid energy cost reduction resulting in electric bill savings. In this context, we apply the proposed heuristic online battery management algorithm described in [18], that requires only the current state of battery level and electricity price to decide whether to use or store RE. Table III summarizes the decisions for different cases.

TABLE III: SPAEMA decisions for different cases of battery and electricity price.

\begin{tabular}{|c|c|c|c|}
\hline Battery/price & Low & Medium & High \\
\hline \hline Low & store & store & use \\
\hline Medium & store & use & use \\
\hline High & use & use & use \\
\hline
\end{tabular}

\section{On-Grid Network Power Consumption}

Following the power model described in eq. (1) and based on the above mentioned algorithms (sleep scheme, RB allocation and battery power management, SPAEMA), we can express the on-grid energy consumption of the network at time stage $i$ as follows:

$$
\begin{aligned}
G_{i}= & \sum_{j=1}^{M}\left(\overline{S D}_{j}^{(i)} \times N_{\text {trx }}\left(P_{0}+\frac{n_{R B}^{(i)}}{n_{R B}^{\text {Total }}} \times \Delta_{p} \times P_{\text {out }}^{(i)}\right)\right. \\
& \left.+S D_{j}^{(i)} \times P_{\text {sleep }}-R E_{j}^{(i)} \times E_{j}^{(i)}\right)
\end{aligned}
$$

where $E_{j}^{(i)}$ is the amount of harvested energy allocated at time stage $i . S D_{j}^{(i)}, \overline{S D}_{j}^{(i)}$ and $R E_{j}^{(i)}$ are binary variables. $S D_{j}^{(i)}$ is a switching decision variable that indicates whether BS $j$ is active $\left(S D_{j}^{(i)}=0\right)$ or in sleep mode $\left(S D_{j}^{(i)}=1\right) . \overline{S D}_{j}^{(i)}$ is the complement of $S D_{j}^{(i)}$. $R E_{j}^{(i)}$ indicates whether BS $j$ is equipped with RE source or not $\left(R E_{j}=1\right.$ indicates that $\mathrm{BS} j$ is equipped with RE source). From eq. (5), a trade-off exists between the number of sites equipped with RE sources and the size of the candidate set of BSs allowed to switch to sleep mode, in order to minimize the energy consumption. In other words, when the number of sites equipped with RE sources increases, less BSs will be able to switch off to save energy; however, more green energy will be introduced into the system to minimize the on-grid energy consumption. In the next section, we demonstrate the trade-off between the percentage of sites equipped with RE sources and the operational cost savings.

\section{Simulation AND Results}

To evaluate the performance of the network, we develop our simulation results via Monte-Carlo method. For each scenario, we run 100 independent simulations using MATLAB and acquire the average value. We consider the use of solar panels, due to their flexibility, and model the variation of electricity price following a real time price (RTP) model, showing the price variation in France during a workday, as shown in Fig. 2. We model the path loss as described in [19], with a carrier frequency of $2 \mathrm{GHz}$ and $\mathrm{W}=10 \mathrm{MHz}$ bandwidth: 


$$
\begin{aligned}
L_{j}\left(u_{x}\right)(d B)= & 40\left(1-4 \times 10^{-3} \times \mathrm{Dhb}\right) \times \log _{10}\left(\mathrm{~d}_{\mathrm{j}}\left(\mathrm{u}_{\mathrm{x}}\right)\right) \\
& -18 \log _{10}(\mathrm{Dhb})+21 \log _{10}(\mathrm{f})+80 d B
\end{aligned}
$$

where $d_{j}\left(u_{x}\right)$ is the distance in $\mathrm{Km}$ between BS $j$ and user at location $x, \mathrm{f}$ is the carrier frequency in $\mathrm{MHz}$, and Dhb is the BS antenna height in meters, measured from the average rooftop level. We take the antenna height and carrier frequency to be $15 \mathrm{~m}$ and $2 \mathrm{GHz}$, respectively. By adding $\log$-normally distributed shadowing $\left(\log _{10} F=\right.$ $6 d B$ ) to the propagation model, the path loss formula will be reduced to:

$$
\begin{aligned}
h_{j}\left(u_{x}\right)(d B) & =L_{j}\left(u_{x}\right)+\log _{10}(\mathrm{~F}) \\
& =128.1+37.6 \log _{10} \mathrm{~d}_{\mathrm{j}}\left(\mathrm{u}_{\mathrm{x}}\right)+6
\end{aligned}
$$

Table IV summarizes the different parameters and their values used, as well as the assumptions taken in this work.

TABLE IV: Parameters' values and assumptions.

\begin{tabular}{|c|c|}
\hline Parameters & Values \\
\hline \hline Number of BSs & 25 \\
\hline Number of sectors & 3 \\
\hline Bandwidth & $10 \mathrm{MHz}$, FDD \\
\hline $\begin{array}{c}\text { Maximum transmitted } \\
\text { power }\end{array}$ & $43 \mathrm{dBm}$ \\
\hline Inter-cell distance & $1000 \mathrm{~m}$ \\
\hline RB & 50 \\
\hline $\begin{array}{c}\text { Number of users in } \\
\text { the network }\end{array}$ & 400 \\
\hline $\begin{array}{c}\text { User min. required } \\
\text { rate }\end{array}$ & $1 \mathrm{Mb} / \mathrm{s}$ \\
\hline Noise power & $-174 \mathrm{dBm} / \mathrm{Hz}$ \\
\hline$P_{0}$ & $118.7 \mathrm{~W}$ \\
\hline$\Delta_{p}$ & 5.32 \\
\hline PV cell properties & $16 \%$ efficiency \\
\hline Number of stages L & 240 \\
\hline
\end{tabular}

\section{A. Energy and Operational Cost Savings}

We define the operational cost gain as follows:

$$
\text { Operational Cost Gain }=1-\frac{\sum_{i=1}^{L} E_{c}^{g}(i) * p(i)}{\sum_{i=1}^{L} E_{F L}^{g} * p(i)}
$$

where $E_{F L}^{g}$ is the on-grid energy consumption of the network without RE at full load, i.e., using all radio resources. $E_{c}^{g}(i)$ is the on-grid energy consumption at time instant $i$, with RE and using the online energy management strategies described in Section IV. $p(i)$ is the price of electricity at time instant $i$.

We consider several parameters when studying the operational cost gain of the network. In addition to evaluating this gain over different percentage of sites equipped with RE, we investigate the impact of the size of solar panels and the capacity of battery storages on this gain over one day. In Fig. 3, we plot the on-grid energy consumption (Eq. (5)) versus the percentage of sites equipped with RE, for different battery capacity sizes. We observe a linear behavior in the on-grid energy consumption. In order to quantify the cost gain, we plot the operational cost gain (Eq. (8)) in Fig. 4. The $20 \%$ gain obtained when none of the sites are equipped with RE sources and no sleep scheme is considered, is a result of the resource block allocation algorithm, described in Section IV-B, that turns off excess RBs and hence, reduces the energy consumption. We observe that equipping only $30 \%$ of sites with RE sources, achieves a cost saving gain of roughly $58 \%$ while we can reach up to $75 \%$ when all BSs are equipped with solar panels that harvest no more than $35 \%$ of the total energy demand at full load. This amount of harvested energy is related to the panel surface area of $12.5 \mathrm{~m}^{2}$ used in this setup.

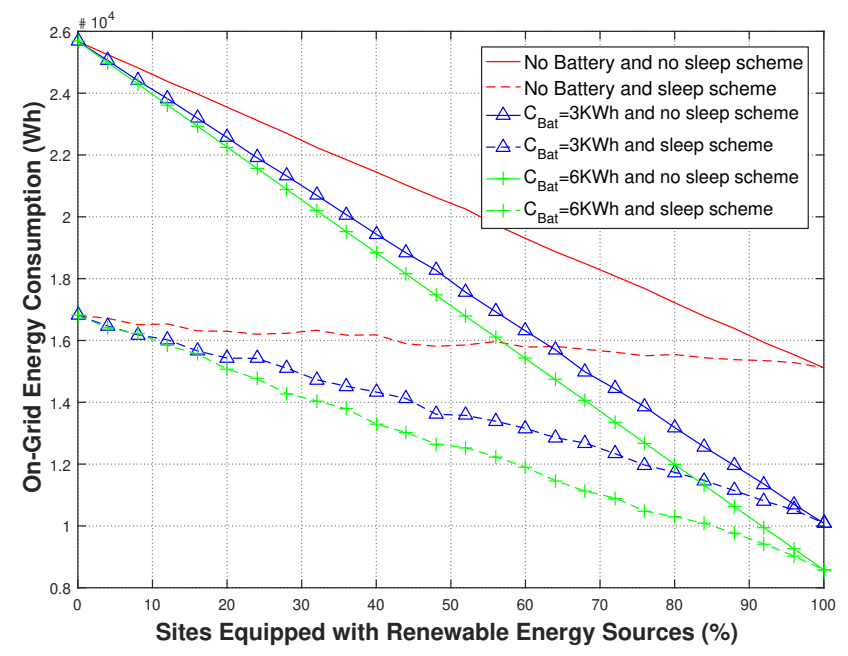

Fig. 3: Network energy consumption over one day, with a $12.5 \mathrm{~m}^{2}$ solar panel surface area.

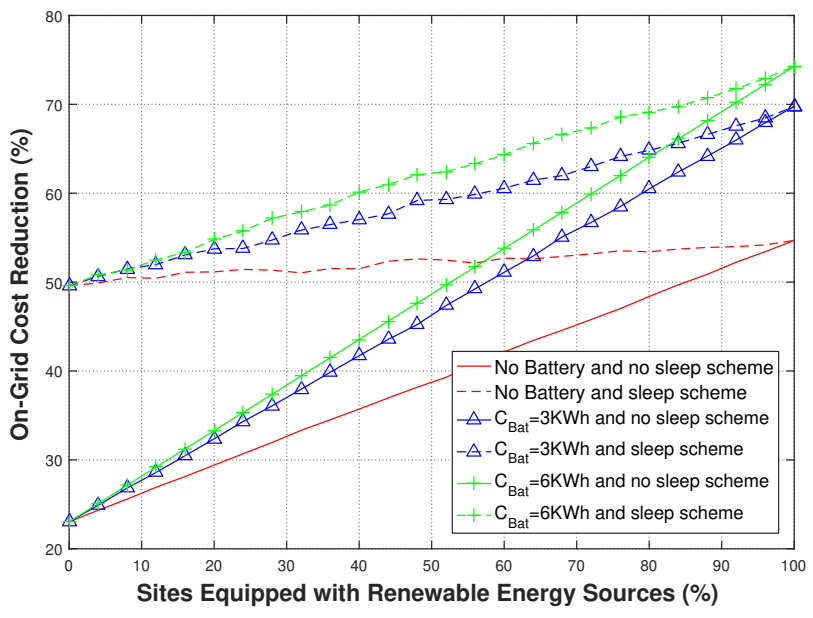

Fig. 4: Percentage of on-grid cost reduction over one day, with a $12.5 \mathrm{~m}^{2}$ solar panel surface area.

Fig. 5 illustrates the operational cost gain versus battery capacity sizes, for different percentage of sites 


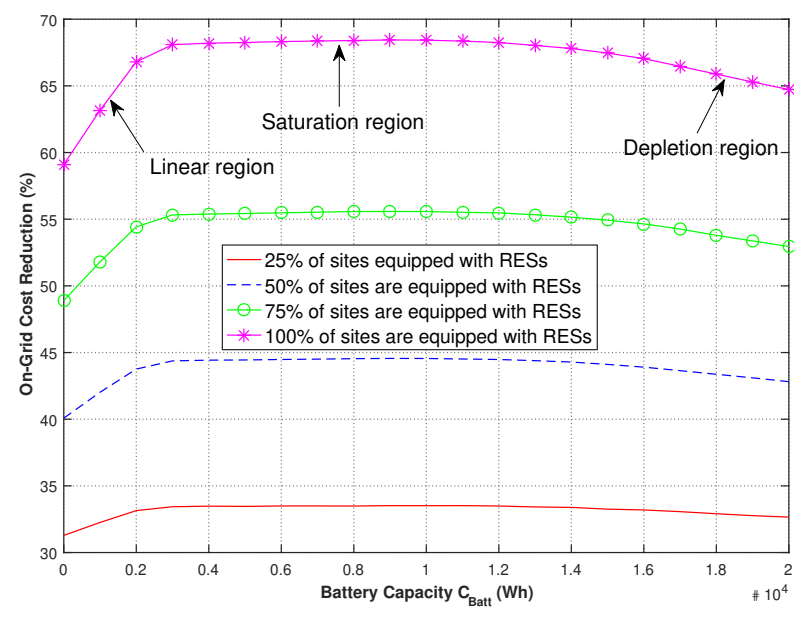

Fig. 5: Percentage of on-grid cost reduction over one day with a $12.5 \mathrm{~m}^{2}$ solar panel surface area, without sleep mode.

equipped with RE sources. We demonstrate the case where sleep mode is not activated, to emphasize the battery capacity impact on the gain achieved solely by RE. The results uncover a cost reduction of around $68 \%$ with a $12.5 \mathrm{~m}^{2}$ solar panel surface area. However, this result is obtained when all the sites are equipped with RE. We also observe that the cost gain is divided into 3 regions. The first region shows a linear increase in the gain versus the battery capacity. As the size of the battery increases, less green energy will be lost due to battery overflow. The second region is denoted as the saturation region. In this region, further increase in the battery capacity brings no significant gain. In this case, the harvested energy is fully used and there is no waste in RE. The last region is the depletion region for large battery capacities. Its behavior is related to the energy management strategy used (here, it is SPAEMA). Based on SPAEMA, if the battery level is low, the harvested $\mathrm{RE}$ will be stored in the battery until it reaches a certain threshold (i.e., medium battery level). However, this threshold increases when the battery capacity is increased. Hence, for large batteries, SPAEMA doesn't properly manage RE and it will keep on storing this energy until this threshold is reached.

Fig. 6 shows the operational cost savings for different solar panel surface areas. Results show that by increasing the size of PV panels, the impact on cost savings from RE increases compared to the savings obtained from sleep scheme only. However, equipping small solar panels with a surface area less than $6 \mathrm{~m}^{2}$ (harvesting less than $10 \%$ of the network total energy demand), degrades the cost savings, compared to the case when using the sleep scheme algorithm described in Section IV-A. This is because solar panels with such harvesting capabilities save less energy than switching off BSs. In this work, we ignore the cost of equipping these sites with RE sources and battery storages. We focus only on the operational cost (OPEX). CAPEX, on the other hand, is left for future work.

Fig. 7 illustrates the cost gain versus $S I N R_{S O T}$. The

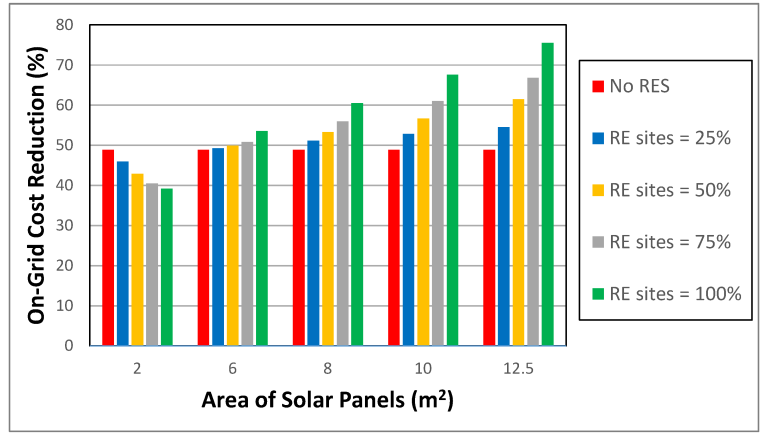

Fig. 6: Percentage of on-grid cost reduction for different panel sizes with sleep mode over one day, with a battery capacity 3 KWh.

results show that as the percentage of sites equipped with RE increases, the impact of $S I N R_{S O T}$ on the cost gain decreases. For instance, when less BSs are equipped with RE sources, more will be able to switch to sleep mode and hence, the SINR switch-off threshold will impact a bigger set of BSs.

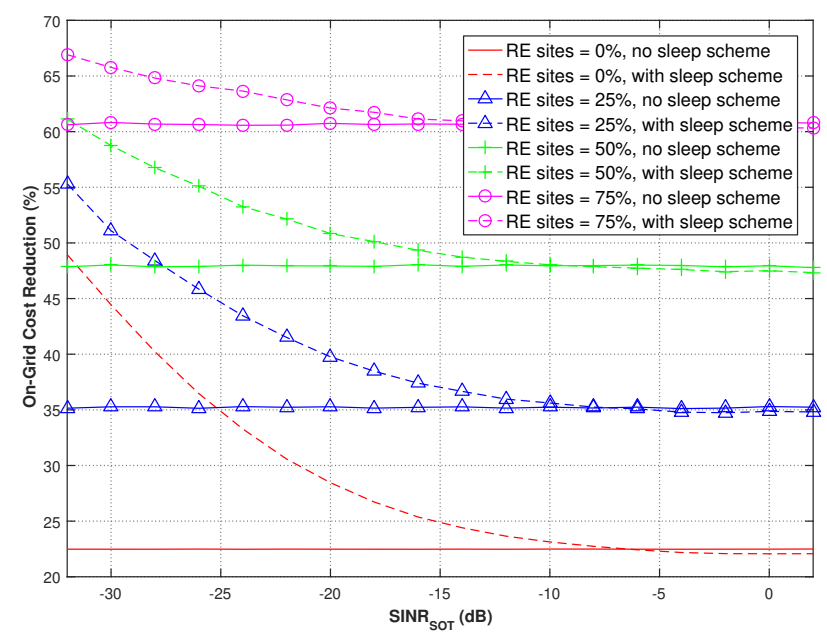

Fig. 7: Impact of the SINR switch-off threshold on the on-grid cost gain, with a $12.5 \mathrm{~m}^{2}$ solar panel surface area and a battery capacity of $3 \mathrm{KWh}$.

\section{B. Network Performance}

Fig. 8 shows the network performance in terms of network's average rate versus $S I N R_{S O T}$. By comparing the results to Fig. 7, a trade-off exists between the cost gain and the QoS, which is defined here as the users' average data rate, when varying the $S I N R_{S O T}$. For example, decreasing the $S I N R_{S O T}$ leads to more cost savings; however, it reduces the user's average rate. We observe that at $-15 \mathrm{~dB}$, the system reaches its peak average rate as some BSs will switch to sleep mode and thus, reducing the interference resulting in a better signal quality. However, at the expense of reducing the cost gain. 


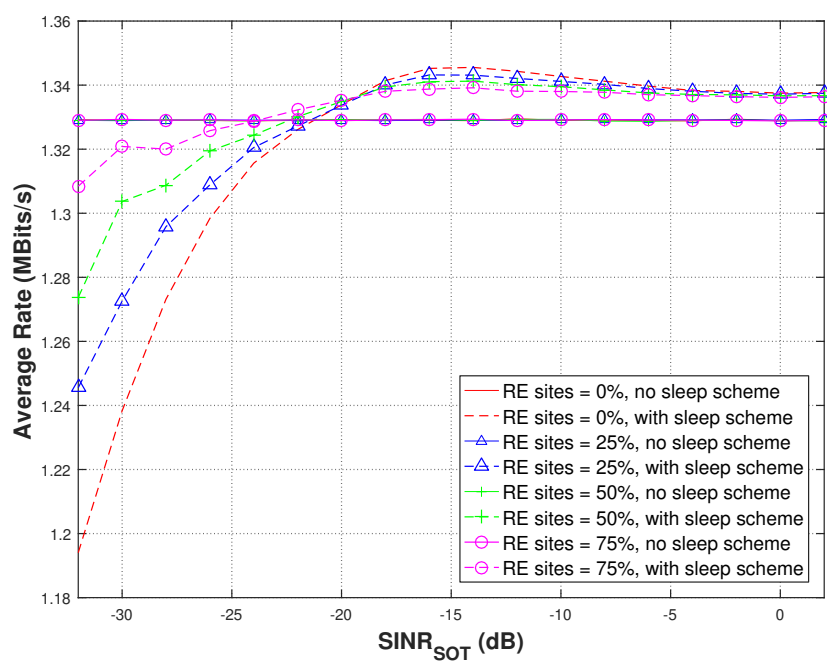

Fig. 8: Network average rate per user for different SINR switch-off threshold, with $12.5 \mathrm{~m}^{2}$ solar panel surface area and a battery capacity of $3 \mathrm{KWh}$.

\section{CONCLUSION}

Using RE in powering BSs in cellular networks introduces significant benefits. In this paper, we demonstrated the effect of equipping BSs with RE sources on the operational cost savings and network performance, for different input parameters: number of sites equipped with RE sources, battery capacity, and solar panel surface areas under different energy efficiency techniques. We reached an operational cost gain of around $60 \%$ when only $30 \%$ of sites are equipped with RE sources. Moreover, we demonstrated a saturation range in which the cost gain does not change by further increasing the battery size. However, for very large battery capacities, the energy management algorithm applied (SPAEMA), fails to reduce the electric bill of the operator. We further studied the trade-off between cost savings and average users' rate by varying the threshold at which BSs can switch to sleep mode. Results show that the impact of this threshold decreases, as the number of sites equipped with RE sources increases.

For future work, we aim at studying more complex algorithms. Using advanced sleep modes to shut down BS's components gradually, is an emerging topic in $5 \mathrm{G}$ networks. Further integration of cellular networks and smart grid can bring additional improvement to the network's energy efficiency. Moreover, contrary to the ideal batteries used in this work, we want to study the system under realistic batteries by considering several indicators such as battery efficiency, battery cycle, depth of discharge and battery lifetime, for different types of batteries.

\section{ACKNOWLEDGMENT}

The authors would like to acknowledge the support of the French Ministry of Industry for supporting this work under SooGREEN project.

\section{REFERENCES}

[1] E. Gelenbe and Y. Caseau, "The impact of information technology on energy consumption and carbon emissions," Ubiquity, vol. 2015, pp. 1:1-1:15, June 2015.

[2] D. Feng, C. Jiang, G. Lim, L. J. Cimini, G. Feng, and G. Y. Li, "A survey of energy-efficient wireless communications," IEEE Communications Surveys Tutorials, vol. 15, pp. 167-178, First 2013.

[3] Cisco Visual Networking Index: Global Mobile Data Traffic Forecast Update, 2016-2021. Cisco, Tech. Rep., February 2017.

[4] M. Etoh, T. Ohya, and Y. Nakayama, "Energy consumption issues on mobile network systems," in 2008 International Symposium on Applications and the Internet, pp. 365-368, July 2008.

[5] L. Suarez, L. Nuaymi, and J.-M. Bonnin, "An overview and classification of research approaches in green wireless networks," EURASIP Journal on Wireless Communications and Networking, vol. 2012, no. 1, p. 142, 2012.

[6] H. A. H. Hassan, L. Nuaymi, and A. Pelov, "Renewable energy in cellular networks: A survey," in 2013 IEEE Online Conference on Green Communications (OnlineGreenComm), pp. 1-7, Oct 2013.

[7] M. Sheng, D. Zhai, X. Wang, Y. Li, Y. Shi, and J. Li, "Intelligent energy and traffic coordination for green cellular networks with hybrid energy supplies," IEEE Transactions on Vehicular Technology, vol. PP, no. 99, pp. 1-1, 2016.

[8] J. Gong, J. S. Thompson, S. Zhou, and Z. Niu, "Base station sleeping and resource allocation in renewable energy powered cellular networks," IEEE Transactions on Communications, vol. 62, pp. 3801-3813, Nov 2014.

[9] H. A. H. Hassan, A. Pelov, and L. Nuaymi, "Cost-efficient radio resource allocation in hybrid energy cellular networks," in 2014 IEEE Global Communications Conference, pp. 2472-2478, Dec 2014.

[10] V. Chamola, B. Sikdar, and B. Krishnamachari, "Delay aware resource management for grid energy savings in green cellular base stations with hybrid power supplies," IEEE Transactions on Communications, vol. PP, no. 99, pp. 1-1, 2016.

[11] Y. Che, L. Duan, and R. Zhang, "Dynamic base station operation in large-scale green cellular networks," IEEE Journal on Selected Areas in Communications, vol. PP, no. 99, pp. 1-1, 2016.

[12] M. Carreno and L. Nuaymi, "Renewable energy use in cellular networks," in 2013 IEEE 77th Vehicular Technology Conference (VTC Spring), pp. 1-6, June 2013.

[13] G. Auer, V. Giannini, C. Desset, I. Godor, P. Skillermark, M. Olsson, M. A. Imran, D. Sabella, M. J. Gonzalez, O. Blume, and A. Fehske, "How much energy is needed to run a wireless network?," IEEE Wireless Communications, vol. 18, pp. 40-49, October 2011.

[14] "PVWatts calculator." last checked: February 2017.

[15] Z. Chen, L. Wu, and Y. Fu, "Real-time price-based demand response management for residential appliances via stochastic optimization and robust optimization," IEEE Transactions on Smart Grid, vol. 3, pp. 1822-1831, Dec 2012.

[16] "Epex, european power exchange spot information." last checked: February 2017.

[17] M. Ghorbanzadeh, A. Abdelhadi, and C. Clancy, Utility Functions and Radio Resource Allocation, pp. 21-36. Cham: Springer International Publishing, 2017.

[18] H. A. H. Hassan, L. Nuaymi, and A. Pelov, "Classification of renewable energy scenarios and objectives for cellular networks," in 2013 IEEE 24th Annual International Symposium on Personal, Indoor, and Mobile Radio Communications (PIMRC), pp. 29672972, Sept 2013.

[19] "Recommendation itu-r m.1225, guidelines for evaluation of radio transmission technologies for imt-2000," 1997. 